\title{
The Early Diagnosis in Lung Cancer by the Detection of Circulating Tumor DNA
}

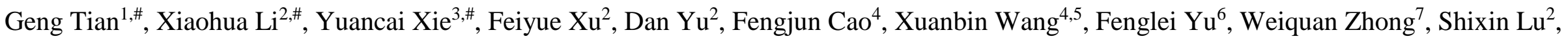
Xiaonian $\mathrm{Tu}^{2}$, Xumei $\mathrm{Yao}^{2}$, Jiankui $\mathrm{He}^{8, *}$, Chaoyu $\mathrm{Liu}^{2}, *$

${ }^{1}$ Department of Oncology, Shenzhen Second People's Hospital, Shenzhen, China

${ }^{2}$ Shenzhen GeneHealth Bio Tech Co., Ltd., Shenzhen, Guangdong, 518053, China

${ }^{3}$ Thoracic Department, Peking University Shenzhen Hospital, Shenzhen, China

${ }^{4}$ Laboratory of Chinese Herbal Pharmacology, Oncology Center, Renmin Hospital, Hubei University of Medicine, Shiyan, 442000, China

${ }^{5}$ Hubei Key Laboratory of Wudang Local Chinese Medicine Research, Shiyan, 442000, China

${ }^{6}$ Department of thoracic surgery, The Second Xiangya Hospital of Central South University, Changsha, China

${ }^{7}$ Department of thoracic surgery, Huizhou Third People's Hospital of Guangzhou Medical University, Huizhou, China

${ }^{8}$ Department of Biology, South University of Science and Technology of China, Shenzhen, 518055, China

\#These authors equally contributed to the manuscript.

*These two corresponding authors equally contributed to the manuscript.

*Correspondence should be addressed to Jiankui He (hejk@sustc.edu.cn) and Chaoyu Liu (liuchaoyu@genehe.com)

Department of Oncology, Shenzhen Second Peoples Hospital, Shenzhen, China 
Background Remarkable advances for clinical diagnosis and treatment in cancers including lung cancer involve cell-free circulating tumor DNA (ctDNA) detection through next generation sequencing. However, before the sensitivity and specificity of ctDNA detection can be widely recognized, the consistency of mutations in tumor tissue and ctDNA should be evaluated. The urgency of this consistency is extremely obvious in lung cancer to which great attention has been paid to in liquid biopsy field. Methods We have developed an approach named systematic error correction sequencing (Sec-Seq) to improve the evaluation of sequence alterations in circulating cell-free DNA. Averagely $10 \mathrm{ml}$ preoperative blood samples were collected from 30 patients containing pulmonary space occupying pathological changes by traditional clinic diagnosis. cfDNA from plasma, genomic DNA from white blood cells, and genomic DNA from solid tumor of above patients were extracted and constructed as libraries for each sample before subjected to sequencing by a panel contains 50 cancer-associated genes encompassing $29 \mathrm{~kb}$ by custom probe hybridization capture with average depth $>40000,7000$, or 6300 folds respectively. Results Detection limit for mutant allele frequency in our study was $0.1 \%$. The sequencing results were analyzed by bioinformatic expertise based on our previous studies on the baseline mutation profiling of circulating cell-free DNA and the clinicopathological data of these patients. Among all the lung cancer patients, $78 \%$ patients were predicted as positive by ctDNA sequencing when the shreshold was defined as at least one of the hotspot mutations detected in the blood (ctDNA) was also detected in tumor tissue. Pneumonia and pulmonary tuberculosis were detected as negative according to the above standard. When evaluating all hotspots in driver genes in the panel, 24\% mutations detected in tumor tissue (tDNA) were also detected in patients blood (ctDNA). When evaluating all genetic variations in the panel, including all the driver genes and passenger genes, 28\% detected in tumor tissue (tDNA) were also detected in patients blood (ctDNA). Positive detection rates of plasma ctDNA in stage I lung cancer patients is $85 \%$, compared with $17 \%$ of tumor biomarkers. Conclusion We demonstrated the importance of sequencing both circulating cell-free DNA and genomic DNA in tumor tissue for ctDNA detection in lung cancer currently. We also determined and confirmed the consistency of ctDNA and tumor tissue through NGS according to the criteria explored in our studies. Our strategy can initially distinguish the lung cancer from benign lesions of lung. Our work shows that the consistency will be benefited from the optimization in sensitivity and specificity in ctDNA detection. 


\section{Introduction}

A growing number of newly diagnosed cancers at the advanced stages are recognized worldwide every year ${ }^{1,2}$, and methods and tools enabling earlier diagnosis are extremely urgent to improve the curative treatment and life quality for cancer patients ${ }^{3}$. Biomarkers consisting of circulating tumor cells, circulating free nucleic acids and exosomes are reported to carry the information of tumorigenesis and provide the clue for early detection of cancers. With the progress of next-generation sequencing (NGS) in recent years, new technology for the detection of these biomarkers called "liquid biopsy" is being developed and attracting more and more attention. Liquid biopsy is expected to substitute solid biopsy in the long term for its noninvasion and convenience in the diagnosis, medication, recurrence monitoring and prognosis assessment of cancers. It is therefore believed to play critical roles in precision medicine and personalized medicine of cancers ${ }^{4,5}$.

Among all the substrates of blood-based liquid biopsy, circulating free DNA (cfDNA) in the blood circulation system has emerged as the most important biomarker because of its availability and stability compared with circulating tumor cells, circulating free RNA and exosomes. The cfDNA in the plasma of cancer patients has been believed to contain circulating tumor DNA (ctDNA) for decades, although over ninety percent of cfDNA in healthy individuals is from metabolism of normal blood cells. Previous studies suggested that cfDNA can serve as a sensitive tool for early diagnosis of cancers ${ }^{6-10}$ and the levels of ctDNA were reported to increase with the severity of cancers ${ }^{11,12}$. Currently, ctDNA is gradually recognized as a significant biomarker for cancer monitoring and treatment ${ }^{3,5}$, especially after several research groups reported the positive detection of ctDNA in early-stagy cancers ${ }^{13-16}$.

However, there are still several difficulties remained to overcome before ctDNA be can widely accepted and used in direct detection of early cancers. Firstly, the amount of ctDNA is limited in blood. The fact that only $0.01 \%-0.1 \%$ of the plasma cfDNA are tumor-related ctDNA and the truth that currently the highest recovery rate of ctDNA is around 10-20\% mean some early cancers are not detectable due to inhesion factor, so technique for ctDNA extraction and enrichment with higher recovery must be developed. Secondly, baseline reference is needed. The removal of the background somatic mutations (the noise) and non-cancer-related genetic variations in cfDNA is technically required for the accuracy in ctDNA detection before it can be steadily used in early cancer diagnosis ${ }^{17}$. Thirdly, consistency should be proved. Namely, genetic variations detected from ctDNA and tDNA (tumor DNA from solid tumor cells) should be proved to be cancer-related. Besides, the evaluating indicator(s) for the assessment of consistency should be reliable and practical.

In order to focus on the above questions, our previous studies explored new resin to increase the recovery rate in ctDNA extraction and examined the background somatic mutations originated from blood cells and cfDNA in 821 non-cancer individuals based on ultra-deep sequencing and we also described the baseline reference ${ }^{18}$. Now we are focusing on the understanding of ctDNA profiles in cancer patients after we developed an updated method called "Sec-Seq" with exogenous molecular barcoding and custom-probe capture to suppress the systematic errors and detect the genetic alterations including mutations, insertion and deletion in early stages of lung cancer. 
Here we report our studies on consistency of ctDNA and tDNA in 30 patients containing pulmonary space occupying pathological changes according to traditional clinic diagnosis. We detected the ctDNA by ultra-deep sequencing for 50 cancer-associated genes encompassing region coverage of $29 \mathrm{~kb}$, proving that the diagnosis based on circulating tumor DNA provided about 50\% and 75\% positive detection rate in phase I and II of lung cancer patients, respectively. Our studies showed the Sec-Seq approach explored in our group can further promote the use of ctDNA in early lung cancer diagnosis and personalized lung cancer therapy.

\section{Methods}

\section{Ethics}

This study was approved by the Shenzhen Second People's Hospital and Peking University Shenzhen Hospital. All the experiments were performed in accordance with guidelines and regulations of the Shenzhen Second People's Hospital and the Peking University Shenzhen Hospital. Written consent was obtained from each patient and all analyses were performed anonymously.

\section{Patients}

A total 30 patients were included in this study with the clinical pathological information in Table S1, which consisted of 2 pulmonary tuberculosis (PTB), 1 organizing pneumonia (OP) and 27 lung cancers including lung adenocarcinoma (LUAD), squamous cell carcinoma (SCC), adenosquamous carcinoma (ASC), sarcoma (SARC) and small cell lung cancer (SCLC). There are three forms including cfDNA, WBC and tissue for each patient. The sample group comprised 33.3\% females and 66.7\% males. The participant ages ranged from 36 to 77 years, with a median of 59.5 years old. Our analysis of the patient clinical information revealed no significant genetic diversity in the samples. The statistical analysis of sample information is summarized in Table $\mathbf{1 .}$

\section{Blood plasma isolation}

For each sample, $10 \mathrm{ml}$ of blood in a cell-free DNA BCT blood collection tube (Streck, Omaha, US) was collected and then centrifuged at 1600 $\mathrm{g}$ for $10 \mathrm{~min}$ at $4^{\circ} \mathrm{C}$ to roughly separate the sample into plasma and blood cells. The upper phase was then transferred into a new tube, leaving around 3-4 mm of "buffering" layer from the buffy coat after the centrifugation and avoid contaminating the plasma layer by blood or cell debris. The plasma was further centrifuged at $16000 \mathrm{~g}$ for another $10 \mathrm{~min}$ to remove the cell debris. The upper clear layer was then aliquoted into 2 -ml tubes, clearly labelled with the patients' identity and immediately stored at $-80^{\circ} \mathrm{C}$ for cfDNA extraction.

\section{Extraction of cfDNA}


Each extraction of cfDNA was performed from $3 \mathrm{ml}$ of plasma. Extraction of cfDNA was conducted using the QIAamp Circulating Nucleic Aid Kit (Qiagen, Hilden, Germany). The concentration of extracted DNA was measured using the Qubit 3.0, dsDNA high-sensitivity assay (Life Technologies, Carlsbad, CA). All methods were performed according the manufacturers' instructions.

\section{Spike in control}

The sensitivity and precision of the current method were evaluated by Horizon's Partners Spike-in control (Horizon, Cambridge, UK) using our custom-designed probes from Integrated DNA Technologies (IDT). Briefly, we performed a serial dilution (from 0.0005 to 1 ) using the wildtype reference genome and the provided reference standard. We performed multiplex PCR, and then library construction and sequenced on the HiseqX10 (Illumina, CA, USA). Six reference variants were included in our primer set: EGFR (L858R, T790M), KRAS (G12D), NRAS (Q61K, A59T) and PIK3CA (E545K). All other primers were used as the background.

\section{Extraction of genomic DNA from WBC}

Genomic DNA of WBCs was extracted by the Qiagen DNA mini kit (Qiagen, Hilden, Germany). The concentration of extracted DNA was measured using the Qubit 3.0, dsDNA high-sensitivity assay (Life Technologies, Carlsbad, CA). All methods were performed according the manufacturers' instructions.

\section{Extraction of genomic DNA from FFPE}

Each extraction of genomic DNA was performed from FFPE. Extraction of genomic DNA was conducted using the QIAamp DNA FFPE Tissue (QIAGEN, Hilden, Germany). The concentration of extracted DNA was measured using the Qubit 3.0, dsDNA high-sensitivity assay (Life Technologies, Carlsbad, CA). All methods were performed according the manufacturers' instructions.

\section{Probe hybridization capture and sequencing library construction}

We developed a probe hybridization capture method to enrich cancer-associated genes. Fifty cancer-associated genes, summarized in Table $\mathbf{2}$, were included and covered a 29139 bp region. A total 2751 mutations were defined as hotspot mutations (Table S2). We performed libraries on circulating DNA from $3 \mathrm{ml}$ plasma using the KAPA kit with standard procedures. We performed libraries on 200ng DNA from WBC or FFPE using the KAPA kit with standard procedures as shown in Figure 1. Barcoding was employed to distinguish real biological mutations from asymmetric DNA errors and sequencing erroes. Indexed libraries were constructed, and hybrid selection was performed with a custom xGen Lockdown Probes Library (IDT) in multiplex. The post-capture multiplexed libraries were amplified with Illumina backbone primers for 16 cycles of PCR using 1× KAPA HiFi Hot Start Ready Mix and then sent to WuxiNextCODE on the Illumina Hiseq X10 platform (Illumina, Beghelli, CA, USA). 


\section{Data filtering and analysis}

We performed ultra-deep target sequencing on 50 cancer-associated genes for both cfDNA and WBC DNA. For each sample, the average sequencing depth was $40000 \times$. The sequencing data was first mapped to the human reference genome (human genome build19; hg19) by Burrows-Wheeler transformation (BWA, Version: 0.7.5a-r405) software package, converted to mpileup format for downstream analysis. We set two filtering criteria to filter the reads: 1) read sequences with mutant allele frequency higher than 5\% in a single read were deleted; and 2) bases with base quality lower than 30 were deleted. Loci with sequencing depth less than $10000 \times$ were removed for further analysis.

\section{Sensitivity evaluation}

The sensitivity and precision of the current method were evaluated by Horizon's Partners Spike-in control (Horizon, Cambridge, UK) using our 207-pair primer set. Briefly, we performed a serial dilution (from 0.0005 to 1) using the wild-type reference genome and the provided reference standard. We performed multiplex PCR, and then library construction and sequenced on the HiseqX10 (Illumina, CA, USA). Six reference variants were included in our primer set: EGFR (L858R, T790M), KRAS (G12D), NRAS (Q61K, A59T) and PIK3CA (E545K). All other primers were used as the background.

\section{Reproducibility evaluation}

To validate the reproducibility of our methods, we drew blood from two healthy individuals, spilt each sample in half, and performed library construction and sequencing independently. We also collected FFPE from two patients, spilt each sample in half, and performed library construction and sequencing independently. By comparing the sequencing data of the two replicates in cfDNA or FFPE samples, we were able to evaluate the stability of the methods and remove background noise. Positions with a sequencing depth lower than $10000 \times$ and mutant allele frequency below 0.001 were excluded in the correlation study.

\section{Mutant allele frequency in cfDNA and WBCs in the 30 patients}

We analysed the correlation of the mutant allele frequency between cfDNA and genomic DNA of WBCs. The mutant allele frequency was defined as mutant allele frequency divided by total reads covering the locus. For example, for a particular position, the total sequencing depth is 10000; we obtain 9990 for A, 3 for C, 5 for G, and 2 for T, and thus the mutant allele frequency for this position is $(3+5+2) / 10000=1 / 1000$. Paired cfDNA and WBCs collected from the same patient (at the same time) were analysed. A total of 29 kb nucleotides in 50 genes were covered in this study. We calculated the average mutant allele frequency of each position in 30 patients in cfDNA and WBCs. We removed the positions with mutant allele frequency higher than 0.1 .

Mutant allele frequency in cfDNA and tDNA in the 30 patients 
We analysed the correlation of the mutant allele frequency between cfDNA and genomic DNA of solid tumor tissue (tDNA). The mutant allele frequency was defined as mutant allele frequency divided by total reads covering the locus. For example, for a particular position, the total sequencing depth is 10000; we obtain 9990 for $\mathrm{A}, 3$ for $\mathrm{C}, 5$ for $\mathrm{G}$, and 2 for $\mathrm{T}$, and thus the mutant allele frequency for this position is $(3+5+2) / 10000=1 / 1000$. Paired cfDNA and tDNA collected from the same patient were analysed. A total of $29 \mathrm{~kb}$ nucleotides in 50 genes were covered in this study. We calculated the average mutant allele frequency of each position in 30 patients in cfDNA and tDNA. We removed the positions with mutant allele frequency higher than 0.1 .

\section{Results}

\section{Validating the sequencing coverage of plasma cfDNA}

The length distribution over all sequences of plasma ctDNA largely fit the normal distribution, with most lengths between 60 to $160 \mathrm{bp}$. Therefore, the sequences in this range of lengths were selected for further analysis (Figure 2A). GC content across all bases of plasma cfDNA was between $45 \%-55 \%$. The region of reads between 1 to 20 bp fluctuated widely and was therefore removed during quality control (Figure 2B) . Evaluation of each amplicon of all plasma samples showed the depth of most amplicons was over 20,000-fold (Figure 2C). Saturation curve suggested the average templates for each plasma sample were around 1800 and dataset of $6 \mathrm{G}$ was sufficient to capture all the information provided in the templates (Figure 2D). Baseline noises of conventional NGS and Sec-Seq after sequencing error corrected are indicated at each base in the captured regions of interest. The noise is lowered by more than 3 orders of magnitude in Sec-Seq (Figure 2E). The quality score of every base called during the sequencing runs illustrated the sequencing accuracy was reliable (Figure S1 A). We also did the validation of tDNA in tumor tissue and validation of gDNA in white blood cells in the initial process of data analysis (Figure S1 B-I).

\section{Validating the detecting sensitivity using the standard reference}

Horizon Standard Reference libraries covering six known tumor-specific hotspot mutations in EGFR, NRAS, PIK3CA and KRAS genes were used to evaluate the sensitivity of the Sec-Seq approach used in cfDNA analysis. The average size of the reference genome is around 160 bp, mimicking the plasma cfDNA that we derived from blood plasma. We examined the sensitivity and precision of this cfDNA reference standard using our sample and library preparation method then tested the sensitivity of the method at variant allele frequencies of $0.0005,0.001,0.005$ and 0.01. Libraries with eight exogenous barcodes were sequenced at an average depth of $>20000$-fold coverage before the allele frequencies detected for six reference mutation sites were calculated. The results demonstrated that our method could detect more than $90 \%$ of variant alleles at a frequency of 0.001 with acceptable fluctuation and the stochastic fluctuation was significantly reduced at the reference 0.005 and 0.01 
(Figure 3). Therefore, threshold of average allele frequency for cfDNA and tDNA detection was subsequently defined as 0.001 , and threshold of average allele frequency for quantitative detection in tumor tissue was defined as 0.02 .

\section{Reproducibility}

We compared the sequencing information of two technical replicate cfDNA samples (left) from the same healthy individual (two individuals in this study) and two technical replicate FFPE samples (right) from the same lung cancer patient (two patients in this study) to evaluate the reproducibility of the Sec-Seq approach in order to distinguish the true biological alterations from systematic errors. Only a total sequencing depth larger than $10000 \times$ and mutant allele frequency larger than $0.1 \%$ for cfDNA samples were included in the correlation study. Our data showed that the mutant allele frequency in the cfDNA samples were highly correlated (Adj $R^{2}=0.8799$ for individual 1 and Adj $R^{2}=0.9589$ for individual 2), implying the reproducibility in plasma ctDNA detection of cancer patients will be even better considering the cfDNA concentration is lower in healthy individuals than in cancer patients (Figure 4). The mutant allele frequency in the two FFPE samples also showed good correlations (Adj $R^{2}=0.9354$ for patient 1 and Adj $R^{2}=0.9448$ for patient 2) (Figure 4), suggesting the Sec-Seq approach can generate satisfactory reproducibilities for all the sample forms in our study (data for white blood cell gDNA not shown).

\section{Consistency between pathology diagnosis and plasma ctDNA mutations detected}

We collected the pre-operation blood and post-operation FFPE samples from 30 patients containing pulmonary space occupying pathological changes according to our experimental design. By using the Sec-Seq approach, we performed ultra-deep target sequencing on 50 cancerassociated genes (Table 2) for plasma DNA (cfDNA) and deep target sequencing for tumor tissue ctDNA, while the white blood cell DNA was also analysed for the somatic mutations in data filtering. Finally, the sequencing data of tumor tissue gDNA for 4 patients were not available The clinic diagnosis was not referred until all the sequencing and technical bioinformatic analysis were completed. The 30 patients turned out to be composed of 2 pulmonary tuberculosis (PTB), 1 organizing pneumonia (OP) and 27 lung cancers diagnosed at stage I to IV (Table 1 and Table S3). We found that the concentration of cfDNA in plasma from cancer patients slightly increased with the progression of cancer, showing average of $20 \mathrm{ng} / \mathrm{ml}$ for stage III patients versus $18 \mathrm{ng} / \mathrm{ml}$ for stage I patients (Table S3), while the trend was not observed for the mutant allele frequency (Table S3 and S4). The ctDNA detection and the diagnosis of SOL of the lung cancer patients demonstrated good consistency between pathology diagnosis and plasma mutations detected. The 3 benign lesion were detected as negative. The overall concordance was $92 \%(\boldsymbol{p}<0.001$, SPSS Statistics version 19). For all the 23 lung cancer patients with qualified sequencing results of ctDNA detection, 85, 80, 100 and 90\% lung cancer patients in stage I, II, III and I-IV, respectively, were predicted to be positive (Table S5).

\section{Comparison of alterations in plasma with those in matched tumor tissue for patients with lung cancer}

The patient characteristics and gene mutations in matched tDNA and plasma ctDNA sample pairs were summarized while patients were categorized based on stage, age, sex, and pathological diagnosis (Table 3 and Figure 5). Only top nine genes were shown here for simplification 
(for detailed information of all the 50 genes in the panel, refer Table S4). Although the sample capacity was limited, it was still found that the consistency in males was better than in females in stage I lung cancer, which may imply the difference of biological situations or responsive abilities between two genders especially in the ultra-early or early stages of cancers as females usually have a higher level of complexity. The consistency rate was evaluated by the percentage of lung cancer patients having one or more shared variants in tumor tissue and plasma (indicated with red star in Figure 5), namely the ratio of the number of patients with ctDNA alterations to the number of patients with the identical alterations in tDNA, which was $78.95 \%$ for the overall I-IV stages (15/19 in Table S5), and 50, 75, 100 or $100 \%$ for patients in stage I, II, III or IV, respectively.

\section{Comparison of ctDNA detection and tumor biomarkers in plasma from patients with lung cancer}

Most of the pre-surgery plasma samples were analyzed for the presence of the following tumor biomarkers including CA125, CA153, CEA, NSE, CA19-9, CYFRA21-1, SCC (squamous cell carcinoma antigen) and AFP (Figure 6 and Table S6) while only the former six were taken into consideration for comparison with plasma ctDNA for the detection of latter two failed in some patients. Finally, there were 22 patients in this analysis. The positive detection was defined as one or more of the above six biomarkers detected as positive. The overall positive rate of plasma ctDNA was $84 \%$ while that of biomarkers was 35\%. More notably, the advantage and significance of plasma ctDNA detection is reflected in early stages in lung cancer. It is obvious that the positive rate of plasma ctDNA for stage I and II was higher than 70\%, when tumor biomarkers were insensitive. 


\section{Discussion}

The ctDNA is released to the peripheral blood in the initiation stage of tumorogenesis, meaning theoretically plasma ctDNA can be detected earlier than CT or other traditional clinical methods in cancer diagnosis. Therefore, cfDNA and ctDNA detection has emerged as the research and commercial frontier of non-invasive cancer biomarkers for monitoring and treatment of cancer ${ }^{19-24}$ in recent years. cfDNA may not $100 \%$ represent the condition of tumor progress, but it is undoubtedly a powerful tool in clinical practice to help diagnose the cancer and evaluate treatment effect to some extent, particularly when the tumor tissue biopsy is hardly feasible. Undoubtedly, the ambition of plasma ctDNA is to promote its use in early diagnosis for cancers with the technical development of NGS and cost decrease of sequencing.

Here we developed a method called Sec-Seq aimed to facilitate the systematic errors correction in ctDNA detection and proved its effectiveness in positive detection of lung cancers, which preliminarily indicates the advantages of plasma ctDNA in diagnosis of early-stage cancers. As a novel probe hybridization capture sequencing technology, Sec-Seq allows ultrasensitive and direct evaluation of sequence changes in circulating cell-free DNA. Although we used it in experiments designed for lung cancer diagnosis, it can be used for any other cancer type.

We previous reported that somatic mutations in the blood cells significantly contribute to mutations in cfDNA in healthy individuals ${ }^{18}$. Now we still noticed this in our current studies (Table S4). These results emphasize again the importance of sequencing both cfDNA and blood cells to remove the background mutations contributed by blood cells.

In our studies, using the Sec-Seq approach, the ctDNA mutations in plasma samples obtained before surgery had a high concordance rate to mutations found in primary tumor tissue, which showed the superiority compared with the detection of tumor markers expression. Besides, the Sec-Seq for cfDNA assay had a satisfactory specificity, sensitivity, and PPV for early diagnosis for patient with SOL of the lung, while these results need to be further confirmed in larger size of samples. Notably, no tumor-derived alterations were identified in the plasma of the patient with benign lesion of the lung in our study.

Our data showed the overall genetic variations (Figure S2) has the comparable concordance between ctDNA and tDNA, most of which are of passenger genes. We believe that more data are required for the elucidation of tumor-related genetic alterations, as the COSMIC hotspots are mainly aimed to target the driver genes.

We also observed the unconsistency between ctDNA and tDNA in our studies (Figure 5) and analysed the ranking of the top 15 genes in our panel in terms of variation probability in patients (Figure 7). It was found that TP53 is the most mutated gene in both ctDNA and tDNA, which has been reported by previous studies. Besides, mutations in TP53, EGFR, PTEN, RET, PIK3CA, KRAS, KDR and ATM were detected in both plasma and tumor tissues in most patients, while the frequencies are partially consistent. We think the cancer heterogeneity of tissue and spacetime of ctDNA may cause the differences. The unconsistency may not mean low sensitivity or false positive result in ctDNA detection, but be originated from the biological reasons including the secretory characteristics, metabolic half-life and cancer heterogeneity. We propose different 
panels should be considered for DNA biomarkers of tumor tissue and plasma cfDNA, since they apparently do not share the ranking of genes in terms of detectable variations.

One more difficulty needs to be pointed out for tDNA mutation detection. We collected the matched FFPE samples but some failed in the DNA extraction or sequencing, resulting in no significant data for 4 lung cancer patients with the reason(s) not determined, which implies the quality control of FFPE sample is very important considering its complexity.

Lastly, we are focusing on a larger sample size to refine the baseline spectrum and more efficient bioinformatics pipeline to increase the accuracy and sensitivity in the mutation detection of ctDNA. More results of our Sec-Seq approach to establish early cancer diagnosis strategies in cancers will be published in upcoming articles. 


\section{Figures and Tables}

Figure 1. Diagram depicting the use of Systematic error correction in ultra-deep sequencing (Sec-Seq) barcode adapters to suppress errors

Figure 2. Sequencing coverage of cfDNA

Figure 3. Sensitivity test using Horizon Reference Standard for cfDNA

Figure 4. Reproducibility validation in cfDNA and tDNA

Figure 5. Consistency analysis of mutations in tDNA and ctDNA

Figure 6. Comparison of positive detection rates of plasma ctDNA and tumor biomarkers

Figure 7. Mutant allele frequency of all the top genetic variations in ctDNA (upper) and tDNA (lower)

Table 1. Clinical features of 30 patients containing pulmonary space occupying pathological changes

Table 2. Genes covered by targeted NGS Panel

Table 3. Consistency analysis of mutations in tDNA and ctDNA

\section{Supplementary data}

Supplementary Figure S1 Sequencing coverage of ctDNA, tDNA or white blood cell gDNA

Supplementary Figure S2 Landscape of genetic alterations in lung cancer patients

Supplementary Table S1 Summary of clinical pathological information

Supplementary Table S2 50-gene panel and probe information

Supplementary Table S3 Relationship between cfDNA centration and stages of lung cancer

Supplementary Table S4 Sequencing data of the 30 patients

Supplementary Table S5 ctDNA detection and the diagnosis of SOL of the lung cancer patients

Supplementary Table S6 Detection of plasma protein biomarkers 
Table 1. Clinical features of 30 patients containing pulmonary space occupying pathological changes

\begin{tabular}{|c|c|}
\hline Characteristic & Parameter value \\
\hline \multicolumn{2}{|l|}{ Age, years } \\
\hline Mean (SD) & $59.5(10.8)$ \\
\hline Median (Range) & $62.5(36-77)$ \\
\hline \multicolumn{2}{|l|}{ Sex, n (\%) } \\
\hline Male & $20(66.7)$ \\
\hline Female & $10(33.3)$ \\
\hline \multicolumn{2}{|l|}{ Pathological diagnosis, n (\%) } \\
\hline non-small cell lung cancer (NSCLC) & $26(86.7)$ \\
\hline Lung adenocarinoma (LUAD) & $19(63.3)$ \\
\hline squamous cell carcinoma (SCC) & $4(13.3)$ \\
\hline small cell lung cancer (SCLC) & $1(3.3)$ \\
\hline ASC with SARC & $1(3.3)$ \\
\hline ASC & $1(3.3)$ \\
\hline SARC & $1(3.3)$ \\
\hline pulmonary tuberculosis (PTB) & $2(6.7)$ \\
\hline organizing pneumonia $(\mathrm{OP})$ & $1(3.3)$ \\
\hline \multicolumn{2}{|l|}{ Tumor stage, $\mathbf{n}(\%$ in all samples) } \\
\hline I A & $7(23.3)$ \\
\hline II A & $2(6.7)$ \\
\hline II B & $4(13.3)$ \\
\hline IIIA & $12(40.0)$ \\
\hline IVA & $1(3.3)$ \\
\hline NA & $4(13.3)$ \\
\hline \multicolumn{2}{|l|}{ Smoking status, $n$ (\% in all samples) } \\
\hline Non-smoker & $14(46.7)$ \\
\hline Smoker & $16(53.3)$ \\
\hline
\end{tabular}


Table 2. Genes covered by targeted NGS Panel

\begin{tabular}{llllllllll}
\hline ABL1 & BRAF & EGFR & FGFR1 & GNAQ & IDH2 & KRAS & NPM1 & PTPN11 & SMO \\
AKT1 & CDH1 & ERBB2 & FGFR2 & GNAS & JAK2 & MET & NRAS & RB1 & SRC \\
ALK & CDKN2A & ERBB4 & FGFR3 & HNF1A & JAK3 & MLH1 & PDGFRA & RET & STK11 \\
APC & CSF1R & EZH2 & FLT3 & HRAS & KDR & MPL & PIK3CA & SMAD4 & TP53 \\
ATM & CTNNB1 & FBXW7 & GNA11 & IDH1 & KIT & NOTCH1 & PTEN & SMARCB1 & VHL \\
\hline
\end{tabular}

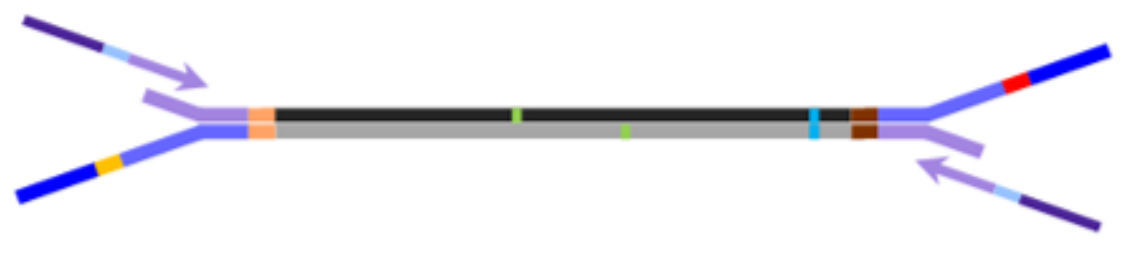

P5

_ Read1 sequence primer

$=+m$ i5 index: N8

P7

Read2 sequence primer

$=\mathrm{i} 7$ index

$=+$ Strand barcode

- Strand barcode A: 6bp

- Strand barcode B: 8bp

- Real biological mutation

. Asymmetric DNA errors

- sequencing errors

Figure 1. Diagram depicting the use of Systematic error correction in ultra-deep sequencing (Sec-Seq) barcode adapters to suppress errors 


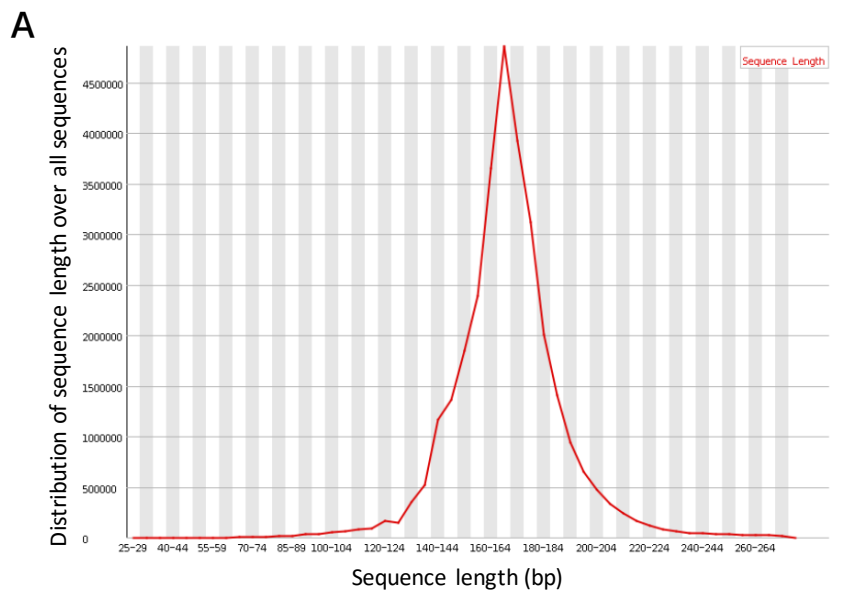

C

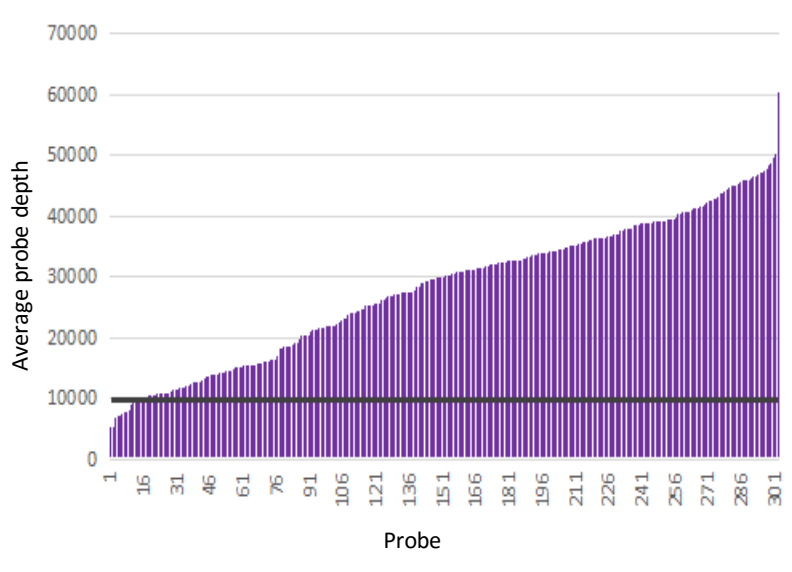

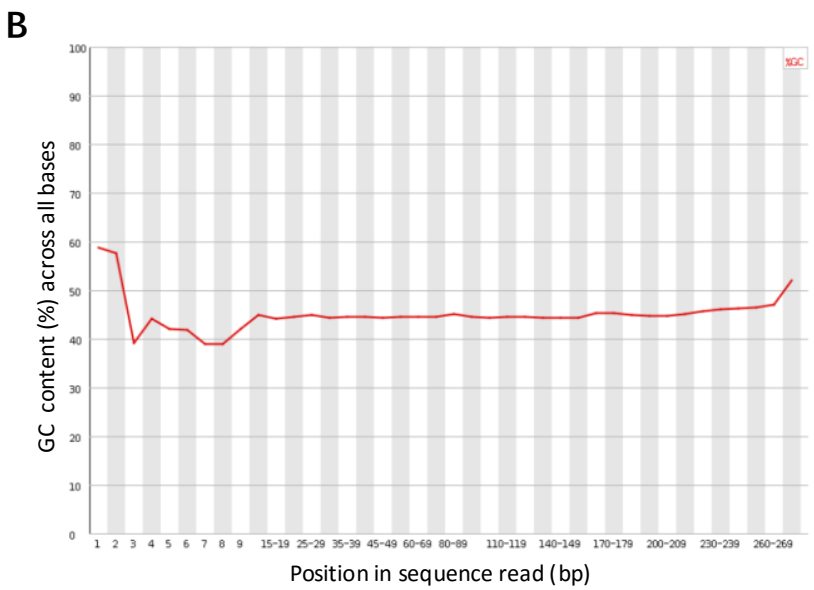

D

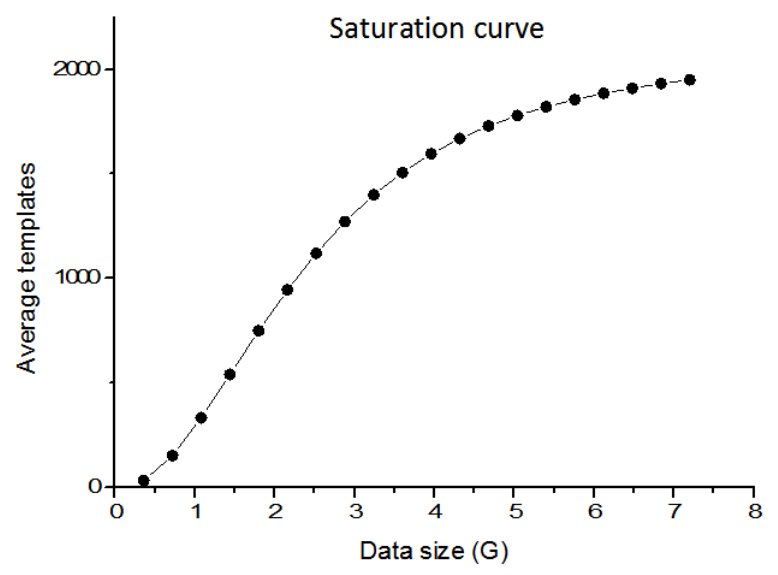

E
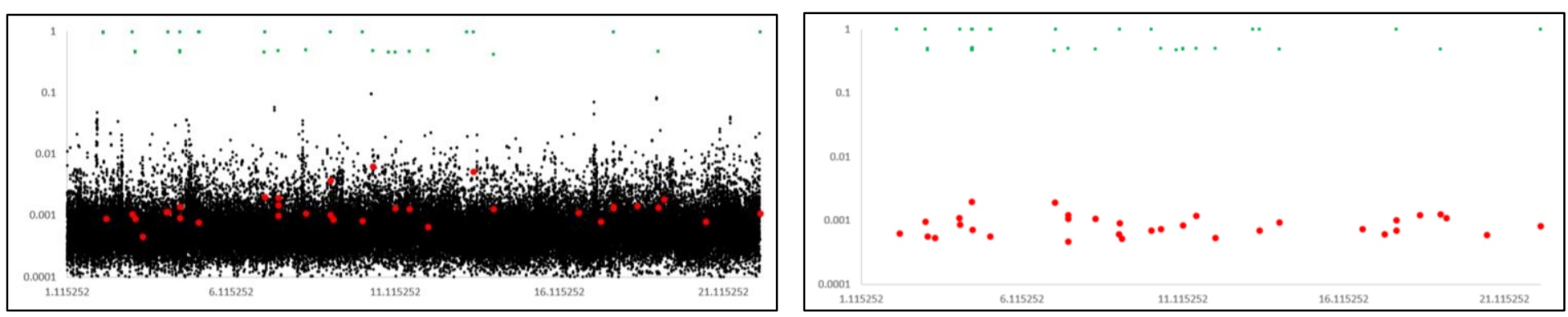

Figure 2. Sequencing coverage of cfDNA. A) Distribution of sequence lengths over all sequences of plasma cfDNA. The sequence length distribution shows most lengths are between 80 to $240 \mathrm{bp}$, which were selected for further analysis; B) GC content across all bases of plasma cfDNA. The region of reads between 1 to 15 bp fluctuates widely and was therefore removed during quality control; $\mathbf{C}$ ) The depth of each amplicon of all plasma samples, where the depth of most amplicons is over 20,000x; (D) The saturation curve shows the average templates were around 1500-2000 with average data set as 5G; (E) SecSeq error correction. 


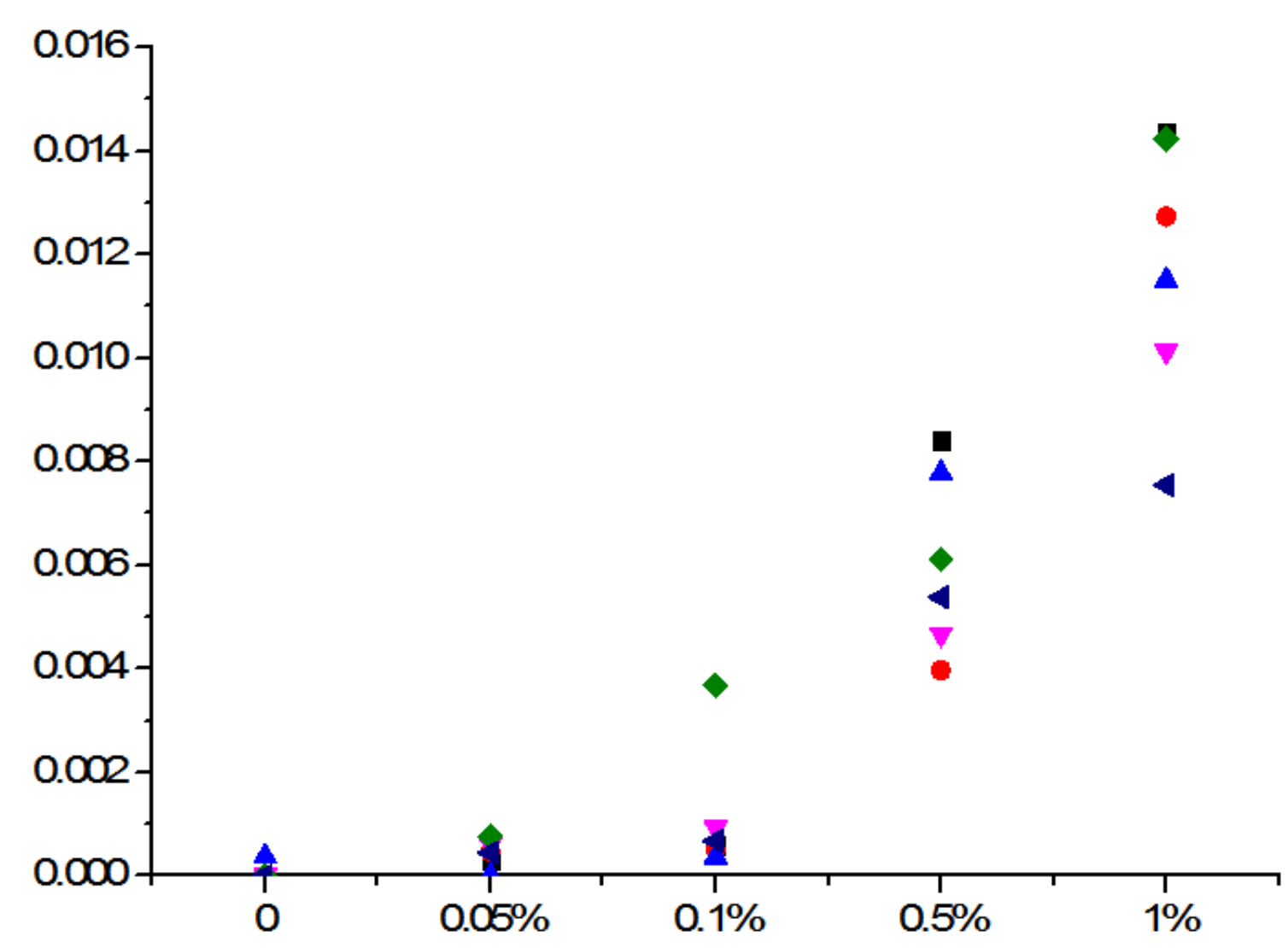

Figure 3. Sensitivity test using Horizon Reference Standard for cfDNA. The reference was manufactured from engineered human cancer cell lines with an allele frequency of $0 \%, 0.05 \%, 0.1 \%, 0.5 \%$ and $1.0 \%$. Deep sequencing was performed and the allele frequencies detected for six reference mutation sites were calculated. 

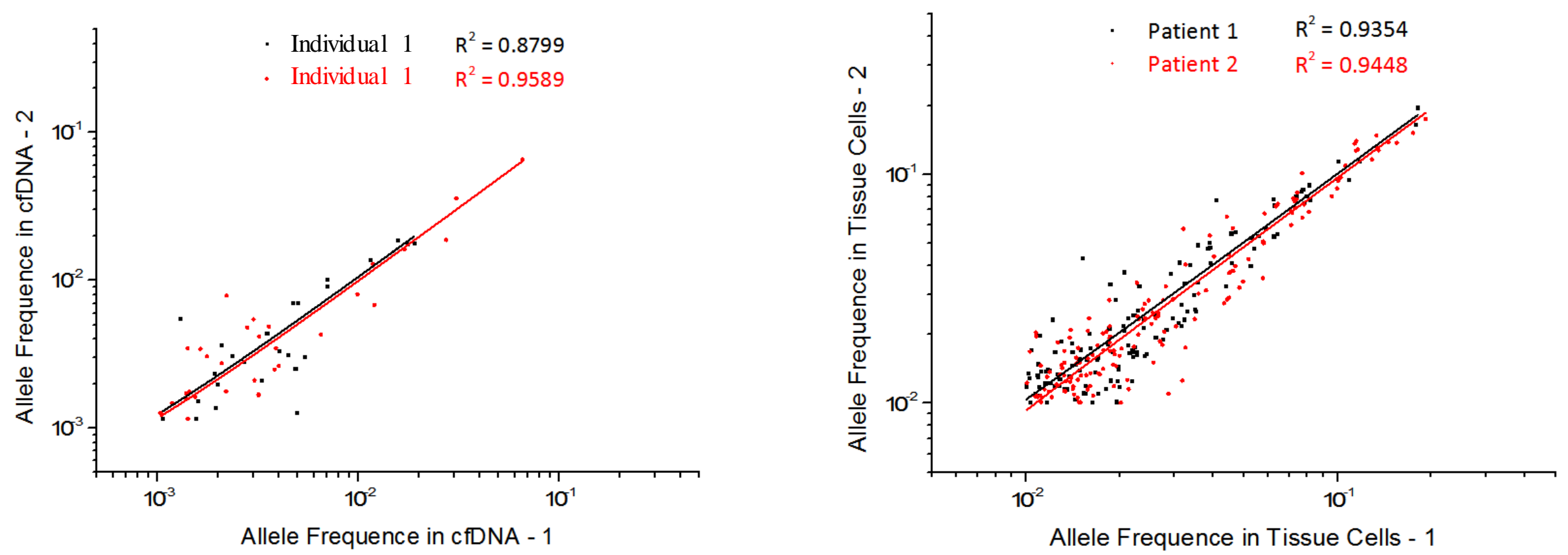

Figure 4. Reproducibility validation in cfDNA and tDNA. We compared the sequencing information of two replicate cfDNA samples (left) and two replicate tDNA samples (right) to evaluate the reproducibility of the Sec-Seq approach. Only a total sequencing depth larger than $10000 \times$ and mutant allele frequency larger than $0.1 \%$ were included in the correlation study. 
Table 3. Consistency analysis of mutations in tDNA and ctDNA

\begin{tabular}{|c|c|c|c|c|c|c|c|c|c|c|c|}
\hline Patient no. & Gene & $\begin{array}{l}\text { Mutation } \\
\text { type }\end{array}$ & AA mutation & $\begin{array}{l}\% \text { mutation in } \\
\text { tDNA (reads) }\end{array}$ & $\begin{array}{c}\text { \% mutation in } \\
\text { pre-op ctDNA } \\
\text { (reads) }\end{array}$ & $\begin{array}{c}\% \text { mutation } \\
\text { in WBC } \\
\text { (reads) } \\
\end{array}$ & $\begin{array}{l}\text { Detected in } \\
\text { tDNA/ctDNA }\end{array}$ & Tumor stage & Age & $\begin{array}{l}\text { Smoking } \\
\text { status }\end{array}$ & Pathological diagnosis \\
\hline \multirow{2}{*}{1} & IDH1 & SNV & p. R132H & $0.037(5400)$ & $0.157(1271)$ & $0.047(10514)$ & $\mathrm{N} / \mathrm{r}$ & \multirow{2}{*}{ Пв } & \multirow{2}{*}{69} & \multirow{2}{*}{ Y } & \multirow{2}{*}{ SCC } \\
\hline & TP53 & SNV & p.R273H & $17.8(4626)$ & $0.118(843)$ & $0.033(9031)$ & $Y / Y$ & & & & \\
\hline \multirow{2}{*}{2} & AKT1 & SNV & p.E17K & $0.031(9583)$ & $0.104(1909)$ & $0(9433)$ & $\mathrm{N} / \mathrm{Y}$ & \multirow{2}{*}{ I A2 } & \multirow{2}{*}{43} & \multirow{2}{*}{$\mathrm{N}$} & \multirow{2}{*}{ LUAD } \\
\hline & GNAS & SNV & p.R201H & $0.131(9127)$ & $0.286(1397)$ & $0.063(11041)$ & $\mathrm{N} / \mathrm{Y}$ & & & & \\
\hline 3 & TP53 & SNV & p.V216M & $19.5(5397)$ & $0.183(2742)$ & $0.076(10409)$ & $Y / Y$ & IIA & 74 & $Y$ & (ASC with SARC) NSCLC \\
\hline \multirow{2}{*}{5} & KRAS & SNV & p.G12V & $26.7(7783)$ & $0.126(793)$ & $0.032(12253)$ & $Y / Y$ & \multirow{2}{*}{ I A3 } & \multirow{2}{*}{53} & \multirow{2}{*}{ Y } & \multirow{2}{*}{ LUAD } \\
\hline & TP53 & SNV & p.R196Q & $0.108(3689)$ & $0.232(861)$ & $\mathrm{O}(12789)$ & $\mathrm{N} / \mathrm{Y}$ & & & & \\
\hline \multirow{2}{*}{6} & EGFR & INDEL & p.L747_P753>S & $28.9(1212)$ & $1.32(1806)$ & $O(16633)$ & $Y / Y$ & \multirow{2}{*}{ ПI } & \multirow{2}{*}{42} & \multirow{2}{*}{$\mathrm{N}$} & \multirow{2}{*}{ LUAD } \\
\hline & TP53 & SNV & p.Q136* & $20.3(531)$ & $0.792(1640)$ & $0.014(13342)$ & $Y / Y$ & & & & \\
\hline & IDH1 & SNV & p. R132H & $0.014(7138)$ & $0.161(1855)$ & $0.059(6682)$ & $\mathrm{N} / \mathrm{Y}$ & & & & \\
\hline 7 & EGFR & SNV & p.L861R & $0.038(10434)$ & $0.296(1687)$ & $0.039(7670)$ & $\mathrm{N} / \mathrm{Y}$ & III & 48 & $\mathrm{~N}$ & LUAD \\
\hline & NRAS & SNV & P.Q61R & $25.7(4929)$ & $\mathrm{O}(1103)$ & $0.528(3783)$ & $\mathrm{Y} / \mathrm{N}$ & & & & \\
\hline 8 & KRAS & SNV & p.G13G & $0.088(10141)$ & $0.128(1561)$ & $0.052(19104)$ & $\mathrm{N} / \mathrm{Y}$ & I A2 & 42 & $Y$ & LUAD \\
\hline 9 & EGFR & INDEL & p.L747_P753>S & $41.8(17443)$ & $\mathrm{O}(3064)$ & $0(6572)$ & $\mathrm{Y} / \mathrm{N}$ & I A2 & 67 & $\mathrm{~N}$ & LUAD \\
\hline 10 & EGFR & INDEL & p.E746_S752>A & $16.7(8544)$ & $\mathrm{O}(1844)$ & $0(8087)$ & $\mathrm{Y} / \mathrm{N}$ & T A & 65 & $N$ & HUD \\
\hline 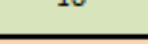 & EGFR & SNV & p.S752F & $18.2(7892)$ & $0.105(1898)$ & $0.012(7740)$ & $Y / Y$ & $1 \mathrm{n}$ & (כ) & v & tom \\
\hline & EGFR & SNV & p.D761N & $23.1(4214)$ & $0.402(1738)$ & $0.031(9602)$ & $Y / Y$ & & & & \\
\hline 11 & TP53 & SNV & p.R273H & $41.7(5756)$ & $2.588(1275)$ & $0.011(8958)$ & $\mathrm{Y} / \mathrm{Y}$ & IIIA & 63 & Y & $\operatorname{scC}$ \\
\hline & AKT1 & SNV & p.E17K & $43.4(5247)$ & $0.125(1598)$ & $0.088(7920)$ & $Y / Y$ & & & & \\
\hline 12 & EGFR & SNV & p.L858R & $40.6(14018)$ & $0.683(1609)$ & & $Y / Y$ & חा & 65 & $y$ & (1) \\
\hline 12 & TP53 & SNV & p.Q192* & $0.214(5137)$ & $0.162(1234)$ & & $\mathrm{N} / \mathrm{Y}$ & m & 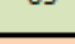 & r & (2) \\
\hline & TP53 & SNV & p.V73L & $1.946(3236)$ & $0.243(2468)$ & & $Y / Y$ & & & & \\
\hline 13 & FGFR3 & INDEL & p.S779fs $* 28$ & $0.125(5588)$ & $0.302(2977)$ & & $\mathrm{N} / \mathrm{Y}$ & IVA & 70 & $\mathrm{~N}$ & LUAD \\
\hline & $\mathrm{IDH} 1$ & SNV & p.R132H & $0.092(8686)$ & $0.117(3418)$ & & $\mathrm{N} / \mathrm{Y}$ & & & & \\
\hline 14 & $\mathrm{KDR}$ & SNV & p.G873R & $18.1(6200)$ & $0.638(1410)$ & $0.020(4986)$ & $Y / Y$ & Пв & 63 & $\mathrm{~N}$ & $(A S C) N S C L C$ \\
\hline - & TP53 & SNV & p.R196Q & $0.234(7688)$ & $0.302(1324)$ & $0.147(4730)$ & $\mathrm{N} / \mathrm{Y}$ & & 等 & & motaves \\
\hline 15 & KRAS & SNV & P.G12A & $29.1(5745)$ & $\mathrm{O}(3353)$ & & $\mathrm{Y} / \mathrm{N}$ & Пв & 69 & Y & LUAD \\
\hline 16 & IDH1 & SNP & p.G105G & $O(3335)$ & $0.140(1419)$ & $0(410)$ & $\mathrm{N} / \mathrm{Y}$ & IIIA & 67 & $Y$ & $\mathrm{SCC}$ \\
\hline & SMARCB1 & SNV & p.? & $11.3(10082)$ & $31.4(1517)$ & & $Y / Y$ & & & & \\
\hline 17 & PDGFRA & SNP & p.V824V & $14.5(5400)$ & $35.6(1754)$ & & $\mathrm{Y} / \mathrm{Y}$ & IIIA & 58 & $\mathrm{Y}$ & SCLC \\
\hline & TP53 & NA & & 0.740404457 & 0.423145401 & & & & & & \\
\hline 18 & TP53 & SNV & p.M237T & $0.068(1461)$ & $0.133(2988)$ & $0.028(7075)$ & $\mathrm{N} / \mathrm{N}$ & $\mathrm{N} / \mathrm{A}$ & 65 & Y & $O P$ \\
\hline 19 & EGFR & SNV & p.L858R & $10.03(13817)$ & $0.846(2362)$ & $0.040(14706)$ & $Y / Y$ & II A & 55 & $\mathrm{~N}$ & PC, (SARC), (NSCLC) \\
\hline & TP53 & SNV & p.R110L & $2.68(5889)$ & $0.375(2133)$ & $0.046(12941)$ & $Y / Y$ & & & & \\
\hline 21 & TP53 & SNV & p.G245S & $0.050(7983)$ & $0.113(1758)$ & $0.0872(18352)$ & $\mathrm{N} / \mathrm{Y}$ & I A & 66 & $\mathrm{~N}$ & LUAD \\
\hline & EGFR & SNV & p.L858R & $20.3(14244)$ & $\mathrm{O}(2183)$ & $0.017(22533)$ & $\mathrm{Y} / \mathrm{N}$ & & & & \\
\hline 22 & PIK3CA & SNV & p. H1047R & $0.091(10908)$ & $3.73(2410)$ & $0.037(29389)$ & $\mathrm{N} / \mathrm{Y}$ & II A & 61 & $Y$ & $S C C$ \\
\hline 2 & TP53 & SNV & p.? & $0.072(12413)$ & $0.622(2570)$ & $0.032(27385)$ & $\mathrm{N} / \mathrm{Y}$ & & & & \\
\hline 23 & KRAS & SNV & p.G12C & $4.66(9002)$ & $O(1738)$ & $0.036(16247)$ & $\mathrm{Y} / \mathrm{N}$ & П & 77 & Y & LUAD \\
\hline 24 & TP53 & SNV & p.V73L & $1.40(2067)$ & $0.213(2814)$ & & $\mathrm{N} / \mathrm{Y}$ & $\mathrm{N} / \mathrm{A}$ & 55 & $Y$ & PTB \\
\hline 25 & TP53 & SNV & p.R202C & $0.120(2486)$ & $0.102(1952)$ & $0.079(17507)$ & $\mathrm{N} / \mathrm{Y}$ & ША & 66 & $\mathrm{~N}$ & LUAD \\
\hline 26 & TP53 & SNV & p.R282W & $0.106(10365)$ & $0.221(905)$ & $0.005(17210)$ & $\mathrm{N} / \mathrm{Y}$ & П $\mathrm{A}$ & 45 & $Y$ & LUAD \\
\hline 27 & JAK3 & SNV & p.? & $0.039(7609)$ & $0.108(1965)$ & $0.028(14164)$ & $\mathrm{N} / \mathrm{Y}$ & IIIA & 36 & $Y$ & LUAD \\
\hline 30 & TP53 & SNV & p.Y234H & $0.066(2986)$ & $0.179(1672)$ & & $\mathrm{N} / \mathrm{Y}$ & $\mathrm{N} / \mathrm{A}$ & 77 & $\mathrm{~N}$ & OP \\
\hline & EGFR & SNV & p.G719A & $8.00(6735)$ & $0(1849)$ & & $\mathrm{Y} / \mathrm{N}$ & & & & \\
\hline
\end{tabular}



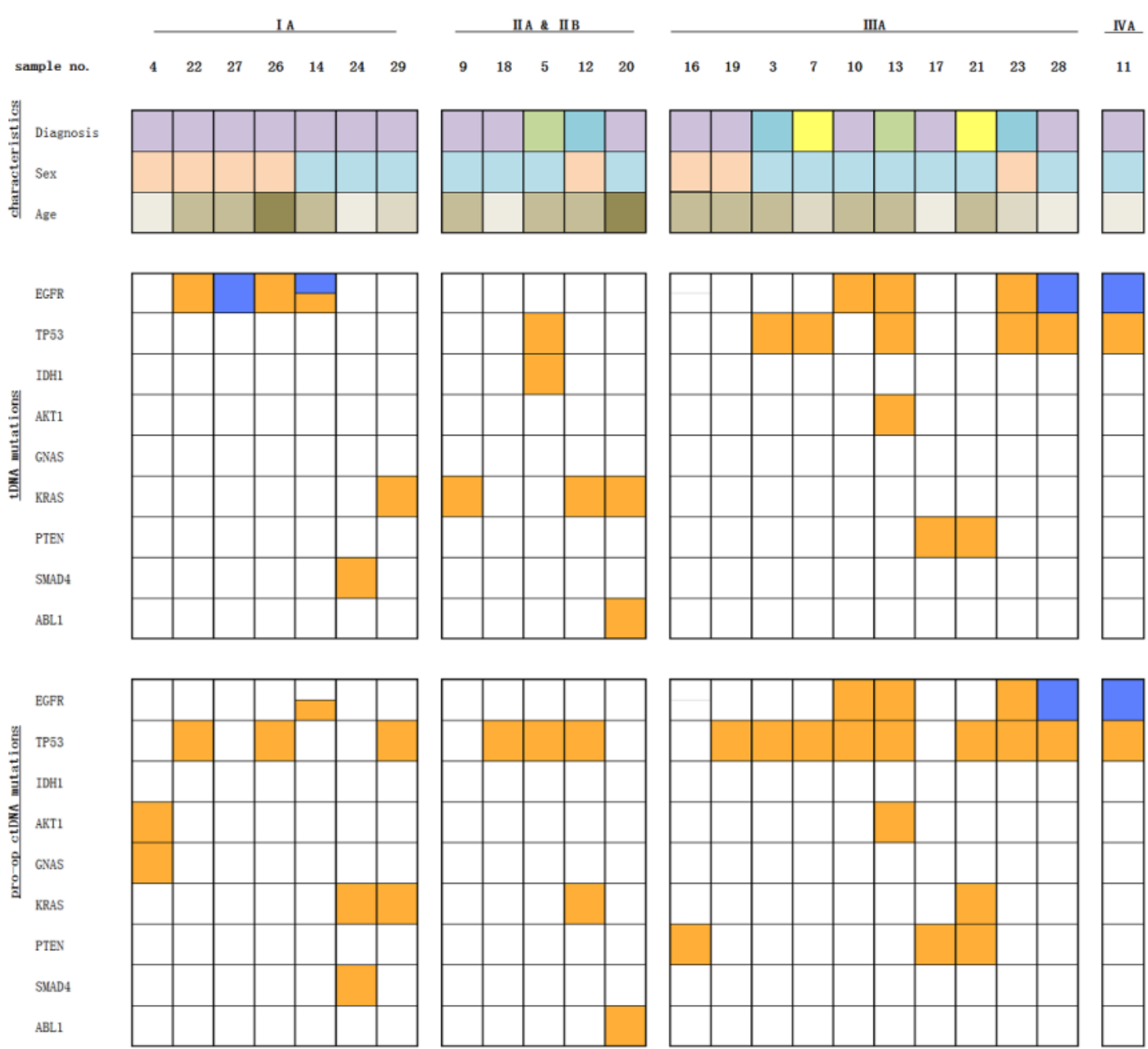

\begin{tabular}{|llll|}
\hline Diagnosis & Sex & Age range(years) & tDNA/ctDNA mutations \\
$\square$ LUAD & $\square$ Male & $\square$ 30-49 & $\square$ sNV \\
$\square$ SCC & $\square$ Female & $\square 50-59$ & $\square$ Indel \\
$\square$ SCLC & & $\square 60-69$ & $\square$ ND \\
$\square$ 0ther NSCLC & $\square 70-79$ & \\
\hline
\end{tabular}

Figure 5. Consistency analysis of mutations in tDNA and ctDNA. Patients were categorized based on stage, age, sex and pathological diagnosis (top); type of mutation per gene in tDNA (middle) and pro-op plasma ctDNA (bottom). tDNA and plasma ctDNA samples with one or more mutations in the same gene are indicated. Orange indicates SNV, blue indicates Indel, and white indicates no genetic variations were detected in that gene in tDNA (middle) or pro-op plasma ctDNA (bottom). Only top nine genes in the panel were shown here for simplification. The consistency rate was evaluated by the percentage of lung cancer patients having one or more shared variants in tumor tissue and plasma. 


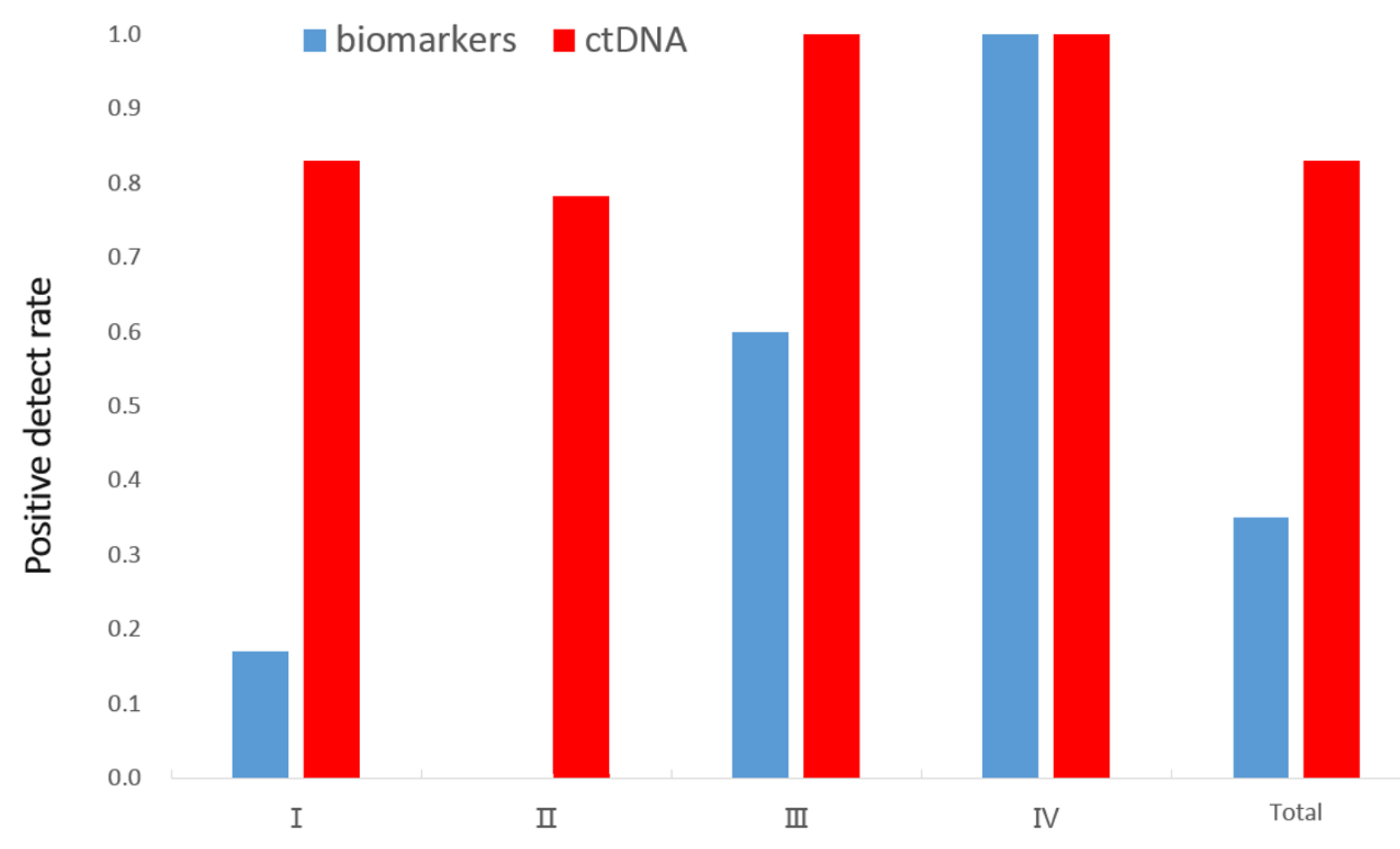

Figure 6. Comparison of positive detection rates of plasma ctDNA and tumor biomarkers. the pre-surgery plasma samples were analyzed for the presence of the following tumor biomarkers including CA125, CA153, CEA, NSE, CA19-9, CYFRA21-1. The positive detection was defined as one or more of these six biomarkers detected as positive. 


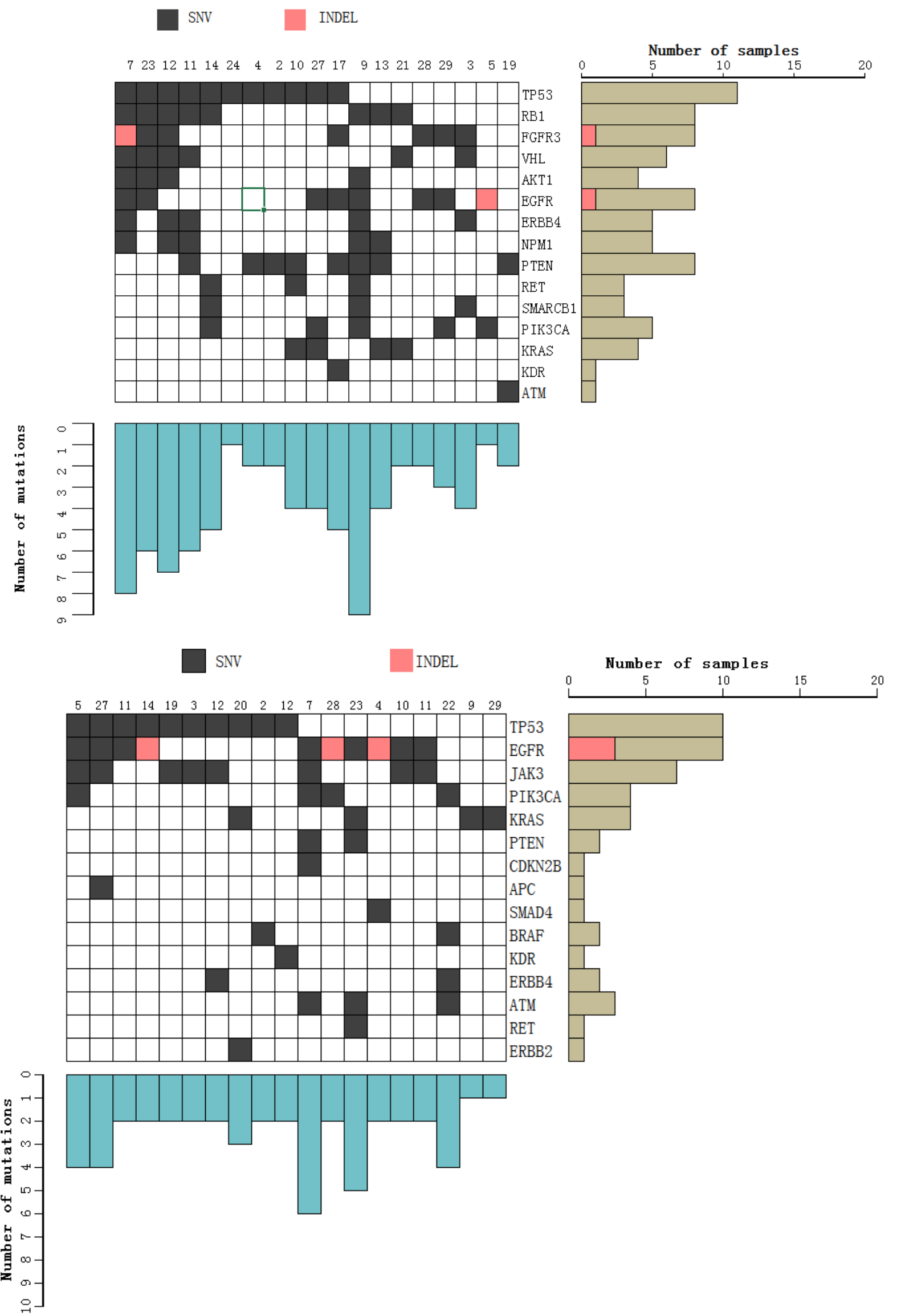

Figure 7. Mutant allele frequency of all the top genetic variations in ctDNA (upper) and tDNA (lower) 


\section{Acknowledgements}

The authors thank Shanghai Mingma for the sequencing services. This work was supported by Shenzhen Innovation Fund of China (Grant No:

CKCY2016082916544973); State Key Research Program of China (Grant No: 2016YFA0501604); Shenzhen Technological Innovation Research

Program of China (Grant No: JSGG20160428090301587); the National Natural Science Foundation of China (Grant No: 31200563); Shenzhen

Basic Research Program of China (Grant No: JCYJ20140819153305695); the Young Scientist Innovation Team Project of Hubei Colleges

(Grant No: T201510); the Key Project of Health and Family Planning Commission of Hubei Province (Grant No: WJ2017Z023).

\section{Author contributions}

J.H., X.L. and C.L. designed the study. G.T., Y.X, F.C., X.W., F.Y. and W.Z. collected original blood samples and tissue samples from the patients. F.X., D.Y. and X.Y. carried out the experiments. S.L. and X.T. summarized and performed the statistical analysis including the clinical information of all the patients. X. L. wrote and revised the manuscript. All authors read and approved the final manuscript.

\section{Competing financial interests}

Xiaohua Li, Feiyue Xu, Dan Yu, Shixin Lu, Xiaonian Tu, Xumei Yao and Chaoyu Liu are employees of Shenzhen GeneHealth Bio Tech Co., Ltd. The other authors declare no competing financial interests. 


\section{References}

Jemal, A. et al. Global cancer statistics. CA: a cancer journal for clinicians 61, 69-90, doi:10.3322/caac.20107 (2011). Torre, L. A. et al. Global cancer statistics, 2012. CA: a cancer journal for clinicians 65, 87-108, doi:10.3322/caac.21262 (2015). von Bubnoff, N. Liquid Biopsy: Approaches to Dynamic Genotyping in Cancer. Oncology research and treatment 40, 409-416, doi:10.1159/000478864 (2017).

Zhang, W. et al. Liquid Biopsy for Cancer: Circulating Tumor Cells, Circulating Free DNA or Exosomes? Cellular physiology and biochemistry : international journal of experimental cellular physiology, biochemistry, and pharmacology 41, 755-768, doi:10.1159/000458736 (2017).

Murphy, D. J. \& Blyth, K. G. Predicting lung cancer recurrence from circulating tumour DNA. Commentary on 'Phylogenetic ctDNA analysis depicts early-stage lung cancer evolution'. Cell death and differentiation 24, 1473-1474, doi:10.1038/cdd.2017.97 (2017). Volik, S., Alcaide, M., Morin, R. D. \& Collins, C. Cell-free DNA (cfDNA): Clinical Significance and Utility in Cancer Shaped By Emerging Technologies. Mol Cancer Res 14, 898-908, doi:10.1158/1541-7786.MCR-16-0044 (2016).

Waldron, D. Cancer genomics: A nucleosome footprint reveals the source of cfDNA. Nat Rev Genet 17, 125, doi:10.1038/nrg.2016.3 (2016).

Togneri, F. S. et al. Genomic complexity of urothelial bladder cancer revealed in urinary cfDNA. Eur J Hum Genet 24, 1167-1174, doi:10.1038/ejhg.2015.281 (2016).

Earl, J. et al. Circulating tumor cells (Ctc) and kras mutant circulating free Dna (cfdna) detection in peripheral blood as biomarkers in patients diagnosed with exocrine pancreatic cancer. BMC Cancer 15, 797, doi:10.1186/s12885-015-1779-7 (2015).

Kienel, A., Porres, D., Heidenreich, A. \& Pfister, D. cfDNA as a Prognostic Marker of Response to Taxane Based Chemotherapy in Patients with Prostate Cancer. J Urol 194, 966-971, doi:10.1016/j.juro.2015.04.055 (2015).

Gonzalez-Masia, J. A., Garcia-Olmo, D. \& Garcia-Olmo, D. C. Circulating nucleic acids in plasma and serum (CNAPS): applications in oncology. OncoTargets and therapy 6, 819-832, doi:10.2147/OTT.S44668 (2013).

Sato, K. A. et al. Individualized Mutation Detection in Circulating Tumor DNA for Monitoring Colorectal Tumor Burden Using a Cancer-Associated Gene Sequencing Panel. PloS one 11, e0146275, doi:10.1371/journal.pone.0146275 (2016).

Phallen, J. et al. Direct detection of early-stage cancers using circulating tumor DNA. Science translational medicine $\mathbf{9}$, doi:10.1126/scitranslmed.aan2415 (2017).

Shu, Y. et al. Circulating Tumor DNA Mutation Profiling by Targeted Next Generation Sequencing Provides Guidance for Personalized Treatments in Multiple Cancer Types. Scientific reports 7, 583, doi:10.1038/s41598-017-00520-1 (2017).

5 Yan, W., Zhang, A. \& Powell, M. J. Genetic alteration and mutation profiling of circulating cell-free tumor DNA (cfDNA) for diagnosis and targeted therapy of gastrointestinal stromal tumors. Chinese journal of cancer 35, 68, doi:10.1186/s40880-016-0131-1 (2016).

Abbosh, C. et al. Phylogenetic ctDNA analysis depicts early-stage lung cancer evolution. Nature 545, 446-451, doi:10.1038/nature22364 (2017).

Martincorena, I. et al. Tumor evolution. High burden and pervasive positive selection of somatic mutations in normal human skin. Science 348, 880-886, doi:10.1126/science.aaa6806 (2015).

8 Xia, L. et al. Statistical analysis of mutant allele frequency level of circulating cell-free DNA and blood cells in healthy individuals. Scientific reports 7, 7526, doi:10.1038/s41598-017-06106-1 (2017).

ctDNA is a specific and sensitive biomarker in multiple human cancers. Cancer discovery 4, OF8, doi:10.1158/2159-8290.CD-

RW2014-051 (2014). 
Kidess-Sigal, E. et al. Enumeration and targeted analysis of KRAS, BRAF and PIK3CA mutations in CTCs captured by a label-free platform: Comparison to ctDNA and tissue in metastatic colorectal cancer. Oncotarget, doi:10.18632/oncotarget.13350 (2016). Ma, F. et al. ctDNA dynamics: a novel indicator to track resistance in metastatic breast cancer treated with anti-HER2 therapy. Oncotarget, doi:10.18632/oncotarget.11791 (2016). doi:10.1038/nrclinonc.2015.159 (2015). Hutchinson, L. Biomarkers: ctDNA-identifying cancer before it is clinically detectable. Nature reviews. Clinical oncology 12, 372 , doi:10.1038/nrclinonc.2015.77 (2015).

Rosell, R. \& Karachaliou, N. Lung cancer: Using ctDNA to track EGFR and KRAS mutations in advanced-stage disease. Nature reviews. Clinical oncology 13, 401-402, doi:10.1038/nrclinonc.2016.83 (2016). 